\title{
Los posibles sí mismos en la adolescencia: diferencias en función del género
}

\section{Possible selves in adolescence: Gender differences}

\author{
María Fernanda Molinaa, ${ }^{\mathrm{a},}$, Melisa Benzi ${ }^{\mathrm{b}}$, Analía Álvarez ${ }^{\mathrm{b}}$ \\ a Universidad Nacional de Tres de Febrero, Argentina \\ bUniversidad de Buenos Aires, Argentina
}

\section{Resumen}

Antecedentes: en la adolescencia, se han encontrado múltiples diferencias en la forma en que los varones y las mujeres se perciben a sí mismos. Esto lleva a preguntarse por la forma en que los y las adolescentes se perciben a sí mismos en el futuro, es decir, los posibles sí mismos (PSs) que pueden generar. Objetivo: indagar las diferencias en función del sexo en los PSs de adolescentes de la Ciudad Autónoma de Buenos Aires (CABA). Método: participaron adolescentes $(n=392)$ de ambos sexos (mujeres $=54.9 \%$ ) que asistían a escuelas secundarias de la CABA (edad $M=14.92 ; S D=1.52$ ). Los adolescentes respondieron un cuestionario de preguntas abiertas en el que se les pidió que realicen un listado de sus PSs cuando terminen el secundario. Se realizó un análisis de contenido temático de las respuestas y conteo de frecuencias. Se utilizaron pruebas de $j i$ cuadrado y U de Mann-Whitney para estudiar las diferencias por sexo en el contenido, diversidad y balance en los PSs. Resultados: las cuatro categorías más mencionadas de PSs por varones y mujeres fueron el estudio, el trabajo, la profesión, el estilo de vida, y la familia y seres queridos. Sin embargo, existen diferencias en la proporción en que varones y mujeres generaron PSs en varios dominios. Por otro lado, las mujeres presentan mayor diversidad de PSs esperados y deseados que los varones. No se encontraron diferencias en el balance en los PSs. Conclusiones: se observa una coincidencia en los dominios de los PSs mencionados por los varones y las mujeres. Sin embargo, las mujeres tienen una mirada más amplia de sí mismas en el futuro y el dominio de la familia y los seres queridos sigue siendo más prevalente para ellas.

Palabras clave: posibles sí mismos, género, adolescencia, autopercepciones.

Para citar este artículo:

Molina, M. F., Benzi, M., \& Alvarez, A. (2019). Los posibles sí mismos en la adolescencia: diferencias en función del género. Liberabit, 25(1), 7-24. doi: https://doi.org/10.24265/liberabit.2019.v25n1.02

\begin{abstract}
Background: in adolescence, multiple differences have been found in the way boys and girls perceive themselves. This leads to wondering about the way in which adolescents perceive themselves in the future, that is, the possible selves (PSs) that they can generate. Objective: to investigate the differences according to sex in the PSs of adolescents of the Autonomous City of Buenos Aires (CABA). Method: the participants were adolescents ( $n=392)$ of both sexes (women $=54.9 \%$ ) who attended secondary schools in the CABA $(M=14.92, S D=1.52)$. The adolescents completed an open-ended questionnaire in which they were asked to make a list of their PSs when they finish high school. A thematic content analysis of responses and a frequency count of categories were conducted. Chi-Square and Mann-Whitney U tests were used to study the differences in content, diversity and balance in the PSs according to sex. Results: the four categories of PSs most mentioned by boys and girls were study, work, profession, lifestyle, and family and loved ones. However, there were differences in the proportion in which males and females generated PSs in several domains. On the other hand, women had more diversity in the expected and desired PSs than men. No differences were found in the balance of the PSs. Conclusions: there is a correspondence between the domains of the PSs mentioned by boys and girls. However, women have a wider view of themselves in the future, and the domain of family and loved ones is still more prevalent in them.
\end{abstract}

Keywords: possible selves, gender, adolescence, selfperceptions.

Este es un artículo Open Access bajo la licencia Creative Commons Atribución-NoComercial-CompartirIgual 4.0

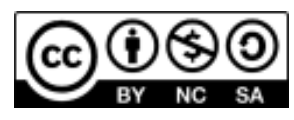




\section{Introducción}

En la adolescencia, se ha encontrado múltiples diferencias en la forma en que los varones y las mujeres se perciben a sí mismos (Oyserman \& Fryberg, 2006). Tradicionalmente, los hallazgos muestran que los hombres y las mujeres difieren en la valencia de sus autopercepciones, es decir, en el grado en que se perciben a sí mismos de manera positiva o negativa. En Argentina, en estudios realizados con niños y preadolescentes, se encontró que las mujeres evidenciaban una autopercepción más positiva de su comportamiento y los varones de la habilidad física (Molina, Raimundi, López, Cataldi, \& Bugallo, 2011). En la adolescencia se observa una autopercepción más positiva de los varones en los dominios de la apariencia física, atractivo amoroso, aceptación social, competencia deportiva y autoestima global (Facio, Resett, Braude, \& Benedetto, 2006). En muestras norteamericanas, se encontraron los mismos resultados para los varones (Harter, 2012).

Por otro lado, el autoconcepto de las mujeres suele ser más influenciado por la relación con sus otros significativos que el de los varones (Oyserman \& Fryberg, 2006). Los hombres son más propensos a tener autoesquemas individualistas, independientes o autónomos. Es decir, los otros son representados como distintos del self. Las mujeres son más proclives a tener autoesquemas colectivistas, ensamblados o conectados, en los que los otros son incorporados como parte del self (Cross \& Madson, 1997; Josephs, Markus, \& Tafarodi, 1992). En Argentina, existen evidencias de que las relaciones con los demás tienen un mayor efecto en el autoconcepto de niñas y adolescentes que en el de los varones (Molina \& Raimundi, 2011; Molina, Raimundi, \& Bugallo, 2017; Molina, Schmidt, \& Raimundi, 2017a; Resett, Meier, García-Sánchez, \& Katz, 2016).

Todas estas diferencias pueden llevan a preguntarse por la forma en que los y las adolescentes se perciben a sí mismos en el futuro, es decir, los posibles sí mismos (PSs) que pueden generar. Este constructo, propuesto por Markus y Nurius (1986), es definido como el autoconocimiento sobre el propio potencial y futuro. Los PSs hacen referencia a cómo los adolescentes piensan que van a ser, cómo temen ser y cómo desean ser en el futuro, aunque no sepan si lo pueden lograr o no.

Los PSs cumplen un rol capital en la exploración de la identidad de los adolescentes (Cadely, Pittman, Kerpelman, \& Adler-Baeder, 2011; Sica, 2009). Asimismo, tienen una importancia central para la elección de acciones entre las posibles conductas y para poder llevar adelante dichas acciones (Oyserman, Bybee, Terry, \& Hart-Johnson, 2004; Shepard \& Marshall, 1999). Sin embargo, no solo importa su contenido. Existen aspectos estructurales que afectan su eficacia motivacional (Oyserman \& James, 2009). Uno de ellos es la disponibilidad, es decir, la facilidad con que pueden ser imaginados. Esta se expresa en el nivel de detalle y elaboración que tienen las descripciones que hacen las personas de sus PSs (Norman \& Aron, 2003). Los PSs específicos y bien elaborados se relacionan con éxito académico futuro y mayor persistencia en la tarea (Leondari, Syngollitou, \& Kiosseogloub, 1998). Otro aspecto estructural de gran importancia es el balance entre los distintos tipos de PSs, es decir, que el individuo posea PSs esperados y temidos en un mismo dominio. De esta forma, se tiene una imagen de qué aspectos del self quiere desarrollar y cuáles evitar en una misma área (Oyserman \& Fryberg, 2006).

En un estudio realizado con adolescentes de la Ciudad de Buenos Aires (Argentina) se encontró que estos proyectaban su visión de sí mismos en el futuro en cinco grandes áreas que agrupaban distintos dominios: sus características personales o rasgos de personalidad; sus ocupaciones y su estilo de vida; su apariencia y aptitudes físicas; sus relaciones interpersonales; y su satisfacción general, aspiraciones y logros. Los PSs más prevalentes eran los referidos a la profesión y ocupación, a los estudios e intereses académicos, y al trabajo. Con respecto a los aspectos estructurales, se encontró que los adolescentes 
presentaban distintos grados de diversidad en el contenido de los PSs que generaban. La mayoría de ellos generó PSs en una o dos áreas de las cinco áreas estudiadas (Molina, Raimundi, \& Gimenez, 2017).

En el estudio mencionado anteriormente, no se consideraron las diferencias en función del sexo. Sin embargo, otras investigaciones encontraron diferencias en los PSs de los y las adolescentes, aunque dichas diferencias no fueron consistentes a través de los contextos y grupos estudiados. Esto plantea la importancia de continuar su estudio (Zhu \& Tse, 2016).

Las diferencias encontradas corresponden a cinco aspectos de los PSs: el número de PSs generados, el contenido más prevalente, la probabilidad percibida de alcanzar PSs positivos, y el balance entre PSs positivos y negativos. A continuación, describimos los resultados encontrados y en la Tabla 1 presentamos un resumen de dichos hallazgos.

Las diferencias en el contenido muestran que las adolescentes generan más PSs temidos sobre funcionamiento interpersonal (Anthis, Dunkel, \& Anderson, 2004; Knox, Funk, Elliott, \& Bush, 2000; McClelland, 2011; Shepard \& Marshall, 1999; Zhu \& Tse, 2016) y el estudio (Zhu \& Tse, 2016). Los varones generan más PSs temidos sobre su ocupación (Knox et al., 2000; Zhu \& Tse, 2016), fracaso general e inferioridad (Knox et al., 2000; McClelland, 2011), comportamiento riesgoso (Zhu \& Tse, 2016) y estilo de vida (Shepard \& Marshall, 1999). En cuanto a los PSs deseados, algunos estudios muestran que las mujeres presentan más PSs en el dominio interpersonal (McClelland, 2011; Zhu \& Tse, 2016) y el estudio (Zhu \& Tse, 2016); y los varones, en el dominio de la carrera profesional y el estilo de vida (Zhu \& Tse, 2016), o las posesiones (Shepard \& Marshall, 1999).

Al considerar las categorías más frecuentemente mencionadas por cada sexo se encontró que los varones formulaban PSs deseados más frecuentemente en el dominio de la ocupación y de las pertenencias y finanzas; mientras que las mujeres lo hacían también en el dominio de la ocupación, pero seguido de las relaciones interpersonales. Los PSs temidos, los PSs de fracaso general, seguidos por los de salud, eran los más prevalentes en los varones. Para las mujeres, los PSs vinculados con el estudio y desarrollo académico eran los más frecuentes, seguidos por las relaciones interpersonales (McClelland, 2011).

Por un lado, en Argentina se ha encontrado que las adolescentes perciben una mayor probabilidad de alcanzar PSs positivos en el dominio de su comportamiento que los varones. Por otro, los varones consideran, en mayor medida que las mujeres, que es probable que puedan tener una habilidad física, apariencia física y un funcionamiento global positivos cuando terminen la secundaria (Molina, Schmidt, \& Raimundi, 2017b). En un estudio realizado con adolescentes norteamericanos se encontró que las adolescentes percibían una mayor probabilidad que los varones de que se realizaran sus PSs temidos, mientras que no hubo diferencias en la percepción de probabilidad de que se realizaran los PSs deseados (Knox et al., 2000). Asimismo, en otro estudio realizado en dicho contexto se encontró que los varones se percibían con más posibilidad de realizar estudios en el área de matemáticas y negocios, mientras que las mujeres en las áreas de humanidades y cultura (Lips, 2004).

En cuanto a los aspectos estructurales, un estudio realizado con adolescentes de Estados Unidos muestra que las mujeres presentan más balance en los PSs interpersonales que los varones (Anthis et al., 2004). Contrariamente, un estudio realizado con población rural de Letonia indica que no se encontraron diferencias (Pavulens, 2013).

Otros estudios muestran diferencias en función del género en el número de PSs generados. En un estudio realizado con adolescentes de Hong Kong, las adolescentes informaron un mayor número de PSs deseados y temidos que los varones (Zhu \& Tse, 


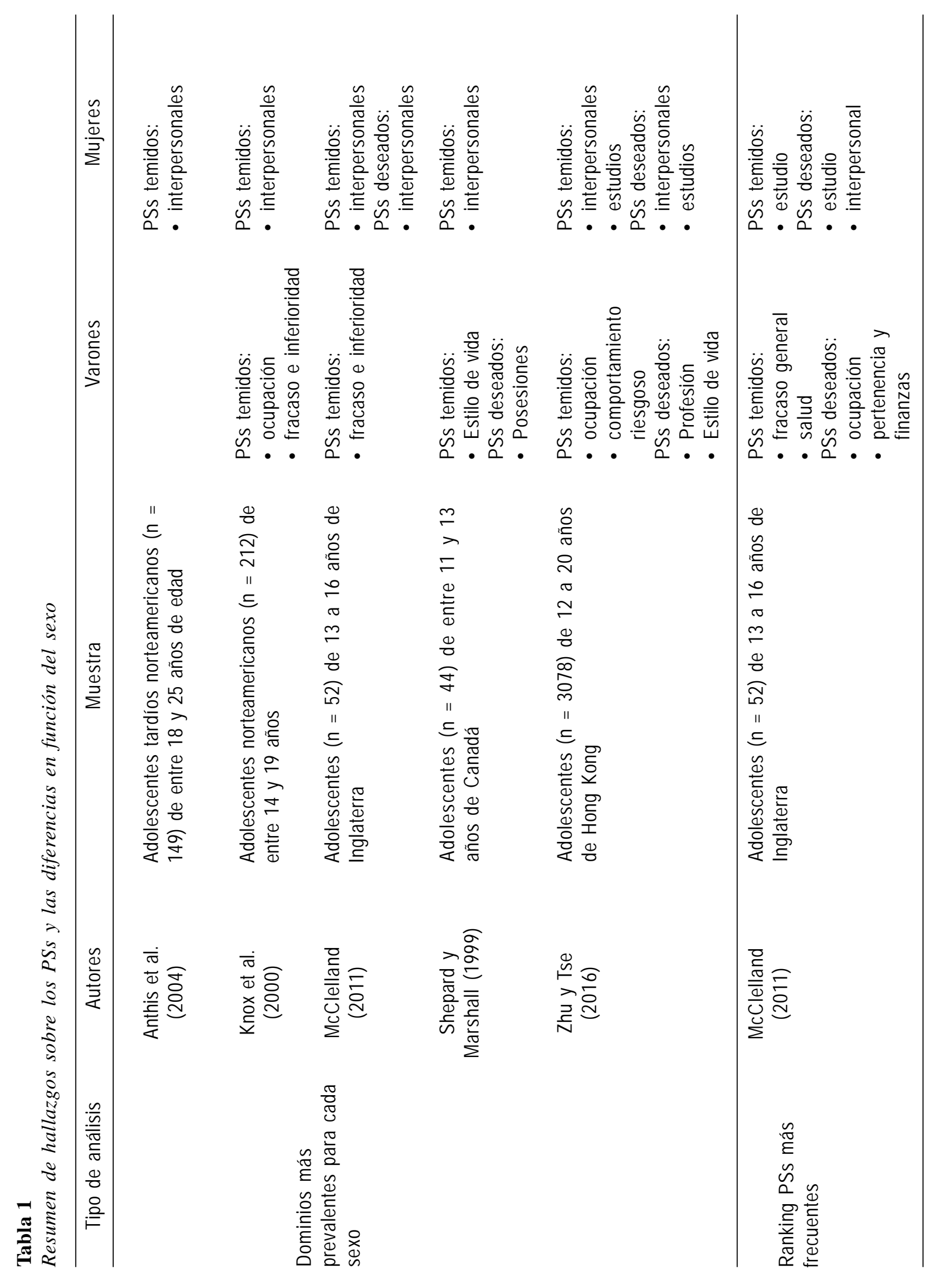




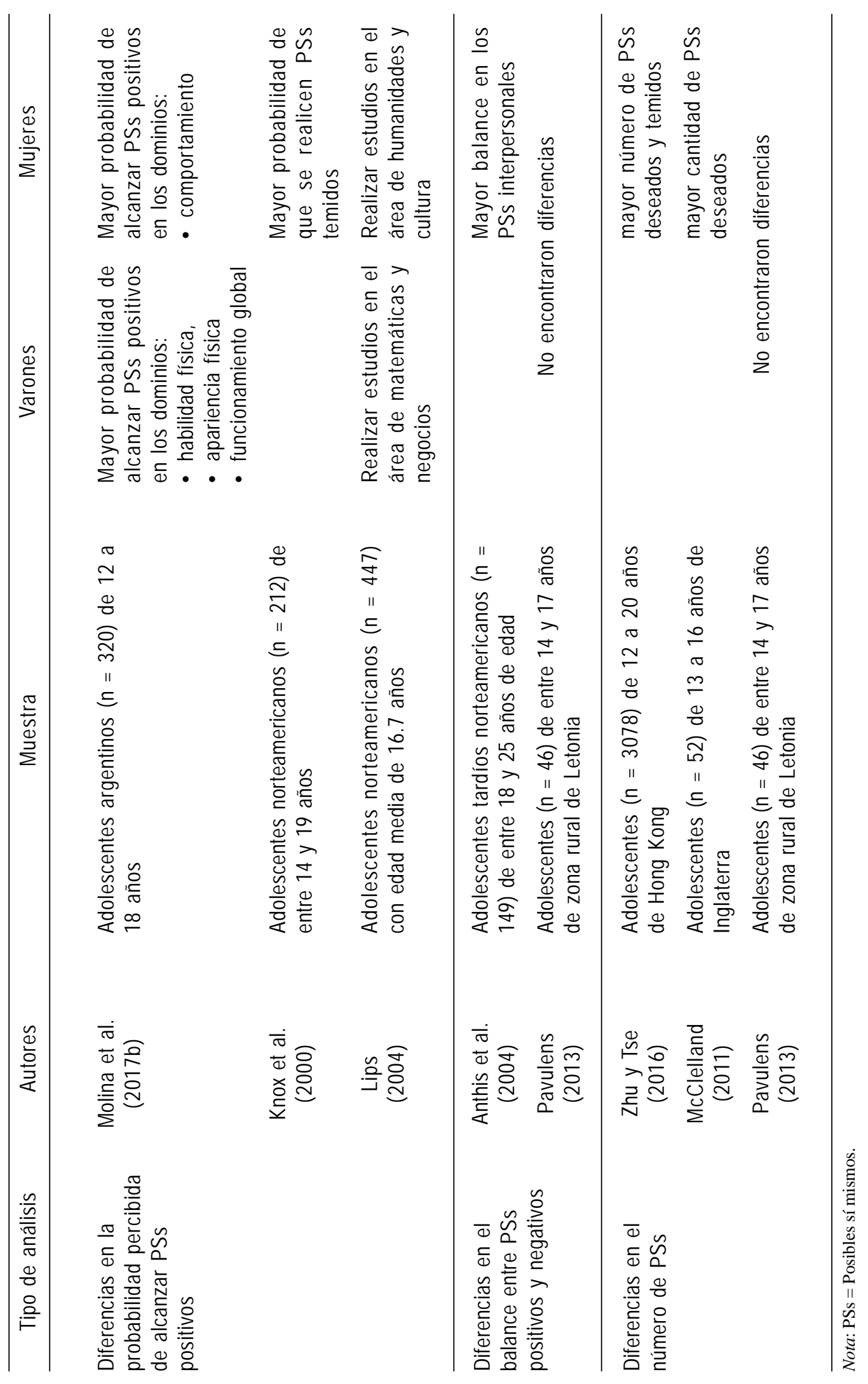


2016). En el caso de los adolescentes norteamericanos se encontró que las mujeres formulaban una mayor cantidad de PSs deseados en comparación a los varones (McClelland, 2011). En cambio, en población rural de Letonia no se encontraron diferencias significativas entre el número de PSs de los y las adolescentes (Pavulens, 2013).

Una cuestión de gran importancia en el estudio de los PSs es el punto de referencia hacia el cual los adolescentes se proyectan. Se pueden crear PSs con una mayor o menor distancia en el tiempo, es decir, los PSs pueden referirse a cómo piensa el adolescente, qué va a ser el próximo año o cómo va a ser en la adultez (Oyserman \& Fryberg, 2006). En particular, consideramos relevante el estudio los PSs de los y las adolescentes al terminar la secundaria. Dicho suceso se encuentra asociado al inicio de la vida adulta. Asimismo, implica una transición vital de gran importancia y el desafío de ingresar en un nuevo contexto (Malin, Reilly, Quinn, \& Moran, 2014). Los estudios citados no indagaron la proyección de los PSs en esta etapa sino en la adultez o en el futuro en general (Molina, Raimundi, \& Gimenez, 2017). Por lo tanto, cabe preguntarse si las diferencias halladas en los PSs de varones y mujeres se mantienen cuando se considera la proyección al final de la secundaria.

Por otro lado, en su mayoría, los estudios antes mencionados se realizaron en países anglosajones. En general, hay una ausencia de estudios sobre los PSs en población latinoamericana (Halfond, Corona, \& Moon, 2013; Unemori, Omoregie, \& Markus, 2004). Si bien en Argentina se ha comenzado a estudiar los PSs, las diferencias en función del sexo necesitan ser estudiadas con mayor profundidad (Molina, Raimundi, \& Gimenez, 2017; Molina et al., 2017b).

Existe gran acuerdo en que los PSs se construyen en las interacciones sociales y están fuertemente condicionados por el contexto cultural e histórico en el que se desenvuelve el individuo. Las representaciones de quién podría ser el adolescente en el futuro están impregnadas por los modelos, imágenes y símbolos que recibe a través de los medios de comunicación y de los microsistemas que habitan (Oyserman \& Fryberg, 2006). Es por esto que es necesario considerar el contenido de los PSs de los adolescentes latinoamericanos, considerando las diferencias entre los sexos.

Según el informe de Desarrollo Humano elaborado por el Programa de Desarrollo Humano de las Naciones Unidas (PDHND, 2018), la Argentina tiene un índice de inequidad de género (GII) de .358. Esto la ubica, en un ranking de 189 países, en el puesto 81. Los países en los que tradicionalmente se han estudiado las diferencias por sexo en los PSs se ubican en puestos superiores. Por ejemplo, Estados Unidos se ubica en el puesto 41; Canadá, en el 20; y el Reino Unido, en el 25. Entre los factores que se utilizan para calcular este índice se consideran: la tasa de mortalidad maternal, tasa de nacimientos adolescentes, proporción de bancas ocupadas por mujeres en el parlamento nacional, porcentaje de la población de 25 años o más que ha alcanzado un nivel secundario de educación, y la tasa de participación en la fuerza de trabajo. En Argentina, se encontró una mayor inequidad en la tasa de participación en la fuerza de trabajo que en otros países. La diferencia entre hombres y mujeres en dicha tasa es del 25.9\%, mientras que en Estados Unidos es del 12.6\%, en Canadá es de 9.1\%, en el Reino Unido de $11.3 \%$ y en China (Hong Kong) es de $14.1 \%$. Sin embargo, en Argentina la proporción de hombres y mujeres con estudios secundarios es equivalente (mujeres 65.9\%, hombres $62.8 \%$ ). Esto es similar en los otros países mencionados.

Los valores de Argentina son similares a los obtenidos por Latinoamérica como región (GII = .386, tasa de mortalidad materna $=67 \%$, tasa de nacimientos adolescentes $=61.5 \%$, mujeres en el parlamento $=28.8 \%$, mujeres con estudios secundarios $=59.5 \%$, hombres con estudios secundarios $=59.1 \%$, mujeres en la fuerza de trabajo $=51.6 \%$, hombres en la fuerza de trabajo $=77.5 \%$ ) (PDHND, 2018). 
Estos datos muestran que pueden existir diferencias en la relación entre los sexos en los distintos países. Estas diferencias pueden derivar en distintos modelos posibles para el self de los y las jóvenes de cada contexto y, por lo tanto, en diferencias en la construcción de sus PSs.

En síntesis, el contenido de los PSs puede variar de acuerdo al contexto social y cultural, así como respecto a la etapa del ciclo vital, el sexo, el grupo étnico, entre otros factores. Esto muestra la importancia de estudiar los patrones de género en los PSs considerando sus especificidades culturales. Este conocimiento es fundamental para poder profundizar la investigación de los PSs o utilizarlos como estrategias de intervención en una nueva población (Zhu \& Tse, 2016).

Por esta razón, este estudio se propone indagar las diferencias en función del sexo en aspectos de contenido, así como también en aspectos estructurales de los PSs de los adolescentes de zonas urbanas de Argentina. Por un lado, nos interesa conocer si existen diferencias en los dominios en los que se proyectan los adolescentes con respecto a las adolescentes. Por otro lado, nos preguntamos si los y las adolescentes difieren en diversidad de dominios en los que pueden crear PSs, a su vez, si existen diferencias en el balance entre los PSs esperados y los temidos.

\section{Método}

\section{Participantes}

La muestra estuvo compuesta por adolescentes $(n=392)$ de ambos sexos (mujeres $=54.9 \%)$ que asistían a escuelas, privadas y católicas, de enseñanza media de la Ciudad de Buenos Aires (edad $M=14.92$; $S D=1.52)$. Un $64.9 \%$ de los adolescentes pertenecían a hogares biparentales. La mayoría de los padres estaban casados entre sí (madres $=69.2 \%$, padres $=69.8 \%$ ). Un $63.2 \%$ de las madres y el $62.0 \%$ de los padres tenía estudios universitarios completos. La mayoría de los padres tiene un empleo estable (madres $=86.2 \%$, padres $=93.6$ ).

\section{Instrumentos}

Cuestionario socio-demográfico. Cuestionario especialmente diseñado para caracterizar a la muestra en función de variables sociodemográficas (e.g., edad, nivel educativo de los padres, composición familiar).

Cuestionario de los posibles sí mismos (Oyserman, 2004). Es un cuestionario de preguntas abiertas en el que se pide al adolescente que realice un listado de cuatro posibles sí mismos esperados, deseados y temidos. A continuación, se detalla la consigna dada a los adolescentes:

«Todos tenemos imágenes e ideas sobre cómo seremos en el futuro, sobre la clase de persona en la que nos convertiremos. ¿Te imaginás cómo serás cuando termines la secundaria?

\section{En los renglones de abajo escribí cómo creés que vas a ser cuando termines la secundaria.}

Además de las imágenes de cómo vamos a ser, todos tenemos ideas de cómo NO queremos ser o de cómo TEMEMOS ser. Pensá un momento en cómo NO querés ser cuando se termine la secundaria. Escribilo en los renglones que están debajo.

Además de cómo creemos que vamos a ser o cómo no queremos ser, hay cosas que deseamos ser, sin importar si es muy probable o muy poco probable que podamos ser así. Por ejemplo, podemos desear ser buenos alumnos o podemos desear ser campeones olímpicos.

Pensá en cómo deseas ser cuando termines la secundaria y escribilo en los reglones que están debajo.»

\section{Procedimiento}

Se contactó a las autoridades de la escuela en la que se realizó el estudio y se les explicó el propósito del proyecto. La participación de los adolescentes fue voluntaria, anónima y confidencial. Se solicitó el consentimiento escrito de los padres indicando que 
aceptaban que sus hijos participaran en el estudio. Los adolescentes respondieron los cuestionarios en forma grupal. La consigna fue explicada por los investigadores.

\section{Análisis de datos}

Se realizó un análisis de contenido temático de las respuestas de los adolescentes, para lo cual se siguieron los lineamientos propuestos por Strauss y Corbin (1990). Tres codificadores analizaron las respuestas de los adolescentes sobre sus PSs esperados. A partir del consenso entre los codificadores se estableció un libro de códigos. Dicho libro de códigos fue aplicado de forma independiente por dos codificadores para analizar las respuestas para los PSs deseados y temidos con un $76 \%$ de concordancia. Se discutieron las categorías en las que no hubo concordancia hasta llegar a un consenso.

Se realizó un conteo de la frecuencia con que las categorías fueron informadas por los y las adolescentes. Para esto se estimó el porcentaje de sujetos que mencionó la categoría, por lo menos, una vez. Para conocer la diversidad del contenido de los PSs expresados por los adolescentes, se calculó la cantidad de dominios a los que hacían referencia los posibles sí mismos de cada adolescente. Para calcular el balance se analizó si los adolescentes generarían PSs con contenido antagónico en los PSs esperados y temidos. Como los adolescentes generaron distinto número de PSs, se calculó el promedio de PSs balanceados respecto del total de pares de PSs.

Se realizaron pruebas chi-cuadrado para estudiar la asociación entre el sexo y la creación de PSs en cada categoría. Se utilizaron pruebas U de MannWhitney para estudiar las diferencias en función del sexo en la diversidad y el balance en los PSs debido a que la distribución de estas variables no es normal.

Para estudiar la magnitud del efecto se utilizó la $r$ de Pearson. Para su interpretación se tomaron los criterios de Cohen (1992) $($ bajo $=.10$; mediano $=.30$; alto $=.50)$. Para estudiar el tamaño del efecto de las pruebas chi-cuadrado se utilizó el coeficiente Phi.

\section{Resultados}

En la Tabla 2 se presentan los porcentajes y las frecuencias observadas con la que varones y mujeres informan PSs en cada dominio. Las pruebas de chicuadrado realizadas indicaron que existe una asociación entre el sexo y la prevalencia de PSs en algunos dominios.

Entre los PSs esperados existe una asociación entre el sexo y los dominios ético/moral $\left(x^{2}[1366]=12.06\right.$, $p=.001, p h i=-.18)$; energía/vitalidad $\left(x^{2}[1366]=\right.$ 3.86, $p=.049$, phi $=.10)$; apariencia física $\left(x^{2}[1366]\right.$ $=6.48, p=.013$, phi $=-.13)$; estilo de vida $\left(x^{2}[1366]\right.$ $=7.62, p=.006, p h i=.14) \mathrm{y}$ bienestar $\left(x^{2}[1366]=\right.$ $6.45, p=.011$, phi $=.13$ ). Como puede observarse en la Tabla 2 existe un mayor porcentaje de varones que refiere PSs en el dominio ético moral y de la apariencia física. En los dominios restantes es mayor el porcentaje de mujeres que construyen PSs que el de varones.

Entre los PSs temidos el sexo se asocia con la presencia de PSs en los dominios: apariencia física $\left(x^{2}[1,370]=5.00, p=.025\right.$, phi $\left.=-.17\right)$, estilo de vida $\left(x^{2}[1,370]=9.55, p=.002\right.$, phi $\left.=-.16\right)$, estudios, aprendizaje y conocimientos $\left(x^{2}[1,370]=9.36, p=\right.$ .002 , $p h i=.16)$, familia y seres queridos $\left(x^{2}[1,370]\right.$ $=6.37, p=.012$, $p h i=.13$ ), confianza en sí mismo $\left(x^{2}[1,370]=7.03, p=.008\right.$, phi $\left.=.14\right) \mathrm{y}$ bienestar $\left(x^{2}[1,370]=4.81, p=.028\right.$, phi $\left.=.11\right)$. Es mayor el porcentaje de varones que informan PSs en los dominios de la apariencia física y estilo de vida. Las mujeres presentan una mayor tendencia a proyectarse en los dominios de la familia y seres queridos, la confianza en sí mismas, el estudio y el bienestar.

Respecto de los PSs deseados se encontró una asociación entre el sexo y los dominios autonomía $\left(x^{2}[1356]=4.87, p=.027\right.$, phi $\left.=.12\right)$; estudios, aprendizaje y conocimiento $\left(x^{2}[1356]=4.70, p=.030\right.$, $p h i=.12)$; $y$ familia y seres queridos $\left(x^{2}[1356]=4.68\right.$, $p=.030$, phi $=.12$ ). En todas las categorías son más las mujeres las que crean PSs. 
Tabla 2

Frecuencias observadas y porcentuales de las categorías de PSs para cada sexo

\begin{tabular}{|c|c|c|c|c|c|c|}
\hline \multirow{2}{*}{ Dominio } & \multicolumn{2}{|c|}{ PSs esperados $n(\%)$} & \multicolumn{2}{|c|}{ PSs temidos $n(\%)$} & \multicolumn{2}{|c|}{ PSs deseados $n(\%)$} \\
\hline & $\begin{array}{l}\text { Mujeres } \\
(n=204)\end{array}$ & $\begin{array}{l}\text { Varones } \\
(n=162)\end{array}$ & $\begin{array}{l}\text { Mujeres } \\
(n=209)\end{array}$ & $\begin{array}{l}\text { Varones } \\
(n=161)\end{array}$ & $\begin{array}{l}\text { Mujeres } \\
(n=200)\end{array}$ & $\begin{array}{l}\text { Varones } \\
(n=156)\end{array}$ \\
\hline Ético/moral & $12(5.9)^{* * *}$ & $28(17.3)^{* * *}$ & $29(13.9)$ & $29(18.0)$ & $19(9.5)$ & 18 (11.5) \\
\hline Responsabilidad & $56(27.5)$ & 32 (19.8) & $54(25.8)$ & $48(29.8)$ & $20(10.0)$ & $7(4.5)$ \\
\hline Energía/vitalidad & $18(8.8)^{*}$ & $6(3.7)^{*}$ & $13(6.2)$ & $4(2.5)$ & $11(5.5)$ & $5(3.2)$ \\
\hline Sociabilidad & $18(8.8)$ & $22(13.6)$ & $42(20.1)$ & $22(13.7)$ & $23(11.5)$ & $15(9.6)$ \\
\hline Competencias & 17 (8.3) & $16(9.9)$ & $12(5.7)$ & $14(8.7)$ & $26(13.0)$ & $16(10.3)$ \\
\hline Confianza en sí mismos & $9(4.4)$ & $2(1.2)$ & $12(5.7)^{* *}$ & $1(.6)^{* *}$ & $7(3.3)^{\mathrm{a}}$ & $1(.6)^{\mathrm{a}}$ \\
\hline Autonomía & $31(15.2)$ & $14(8.6)$ & $32(15.3)$ & $18(11.2)$ & $18(9.0)^{*}$ & $5(3.2)^{*}$ \\
\hline Conducta problemática & - & - & $15(7.2)$ & $21(13.0)$ & - & - \\
\hline Apariencia física & $2(1.0)^{* a}$ & $9(5.6)^{* a}$ & $3(1.4)^{*}$ & $9(5.6)^{*}$ & $6(3)$ & $6(3.8)$ \\
\hline Deportivo & 12 (5.9) & $17(10.5)$ & $4(1.9)^{\mathrm{a}}$ & $3(1.9)^{a}$ & $13(6.5)$ & $19(12.2)$ \\
\hline Estudio e intereses académicos & 118 (57.8) & $78(48.1)$ & $85(40.7)^{* *}$ & $41(25.5)^{* *}$ & $70(35.0)^{*}$ & $38(24.4)^{*}$ \\
\hline Profesión/ocupación & $88(43.1)$ & $73(45.1)$ & $48(23.0)$ & $29(18.0)$ & $97(48.5)$ & $83(53.2)$ \\
\hline Trabajo & $41(20.1)$ & 31 (19.1) & $71(34.0)$ & $67(41.6)$ & $28(14.0)$ & $34(21.8)$ \\
\hline Estilo de vida & $53(26.0)^{* *}$ & $23(14.2)^{* *}$ & $26(12.4)^{* *}$ & $40(24.8)^{* *}$ & $44(22.0)$ & $30(19.2)$ \\
\hline Familia y seres queridos & $42(20.6)$ & 29 (17.9) & $32(15.3)^{*}$ & $11(6.8)^{*}$ & $54(27.0)^{*}$ & $27(17.3)^{*}$ \\
\hline Pares & $17(8.3)$ & $13(7.4)$ & $13(6.2)$ & $7(4.3)$ & $16(8.0)$ & $10(6.4)$ \\
\hline Bienestar & $24(11.8)^{*}$ & $7(4.3)^{*}$ & $22(10.5)^{*}$ & $7(4.3)^{*}$ & $27(13.5)$ & $14(9.0)$ \\
\hline Éxito & $15(7.4)$ & $8(4.9)$ & $13(6.2)$ & $9(5.6)$ & $35(17.5)$ & $17(10.9)$ \\
\hline Metas & $17(8.3)$ & $11(6.8)$ & $26(12.4)$ & $17(10.6)$ & $19(9.5)$ & $9(5.8)$ \\
\hline Intereses políticos y sociales & $11(5.4)$ & $6(3.7)$ & - & - & $6(3.0)$ & $8(5.1)$ \\
\hline Fidelidad con uno mismo & $4(2.0)^{\mathrm{a}}$ & $1(.6)^{\mathrm{a}}$ & $5(2.4)^{a}$ & $2(1.2)^{\mathrm{a}}$ & $3(1.5)^{a}$ & $1(.6)^{\mathrm{a}}$ \\
\hline Ilusiones y sueños & - & - & - & - & $3(1.5)^{a}$ & $2(1.3)^{a}$ \\
\hline
\end{tabular}

Nota: PSs $=$ Posibles sí mismos; ${ }^{*} p<.05 ; * * p<.01 ; * * * p<.001$

a Se utilizó el estadístico exacto de Fisher ya que se más de un $20 \%$ de las casillas tuvieron una frecuencia mínima esperada inferior a 5

En la Tabla 3 se presenta el ranking de categorías más frecuentes para varones y mujeres. Las cuatro categorías de PSs más mencionadas por ambos sexos son el estudio, el trabajo, la profesión, el estilo de vida, y la familia y seres queridos.

En la Tabla 4 se presentan las comparaciones de la diversidad de PSs generados por los y las adolescentes. Encontramos una diferencia estadísticamente significativa en la diversidad de dominios en los PSs esperados y deseados. Es decir, las mujeres crearon PSs deseados y temidos en una mayor cantidad de dominios que los varones. No se observan diferencias en la diversidad de dominios de los PSs temidos. En todos los casos el tamaño del efecto es bajo. 


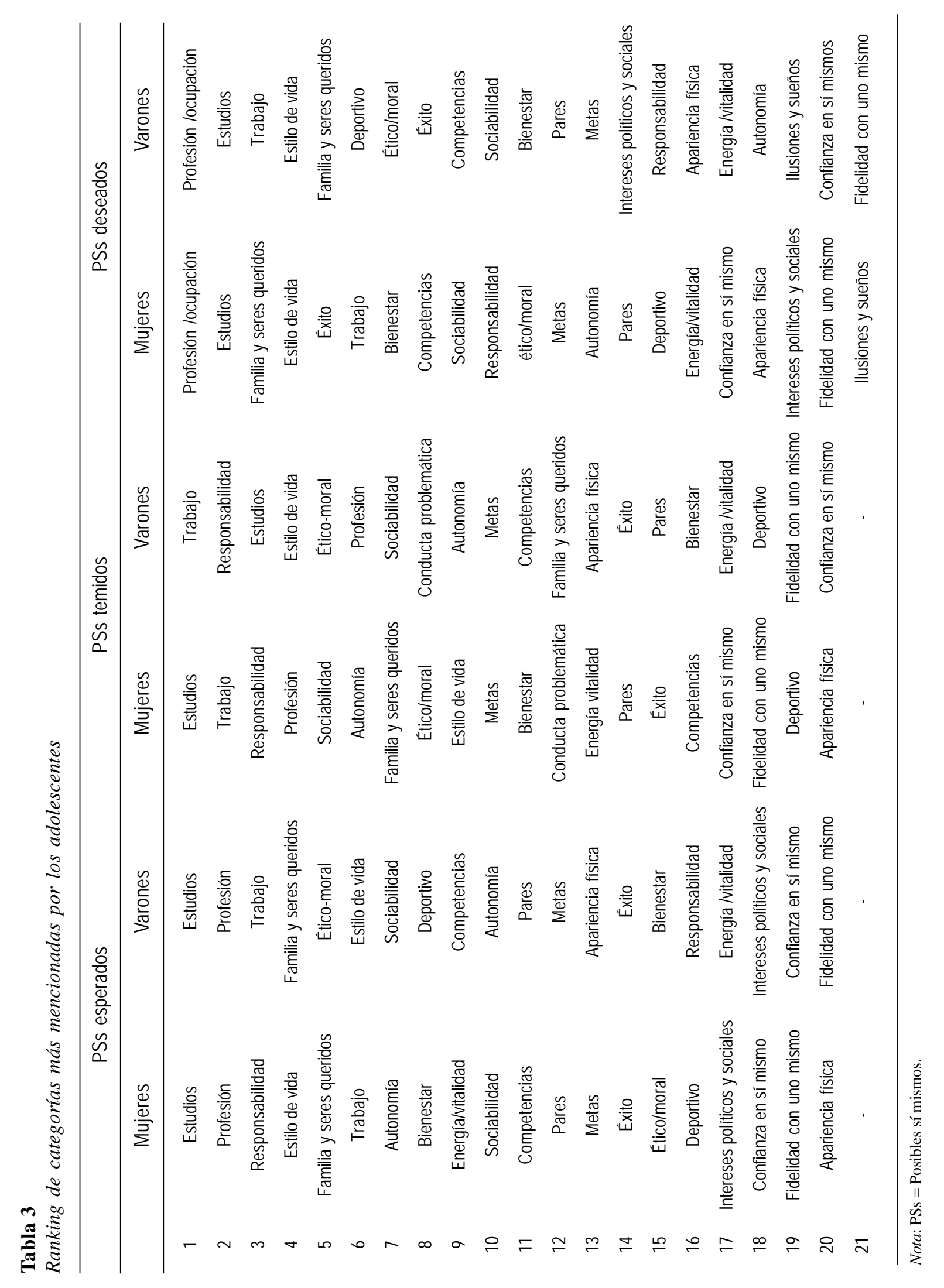




\section{Tabla 4}

Comparación en la diversidad de dominios mencionados por cada tipo de PSs y balance de los PSs esperados y temidos, entre varones y mujeres a través de la prueba U de Mann-Whitney

\begin{tabular}{lcccccc}
\hline & Varones (RP) & Mujeres (RP) & $U$ & $z$ & $p$ & $r$ \\
\hline Diversidad PSs esperados & 169.24 & 194.82 & 14214.000 & -2.353 & .019 & -.12 \\
Diversidad PSs temidos & 175.93 & 192.87 & 15284.000 & -1.558 & .119 & -.08 \\
Diversidad PSs deseados & 159.10 & 193.64 & 12573.000 & -3.238 & .001 & -.17 \\
Balance PSs & 179.67 & 184.72 & 15867.50 & -.46 & .645 & .02 \\
\hline
\end{tabular}

Nota: PSs = Posibles sí mismos; RP = Rango promedio.

Tampoco se encontraron diferencias estadísticamente significativas en el balance de los PSs esperados y temidos de los y las adolescentes. En la
Tabla 5 se presenta un resumen de los resultados encontrados.

\section{Tabla 5}

Resumen de los resultados encontrados

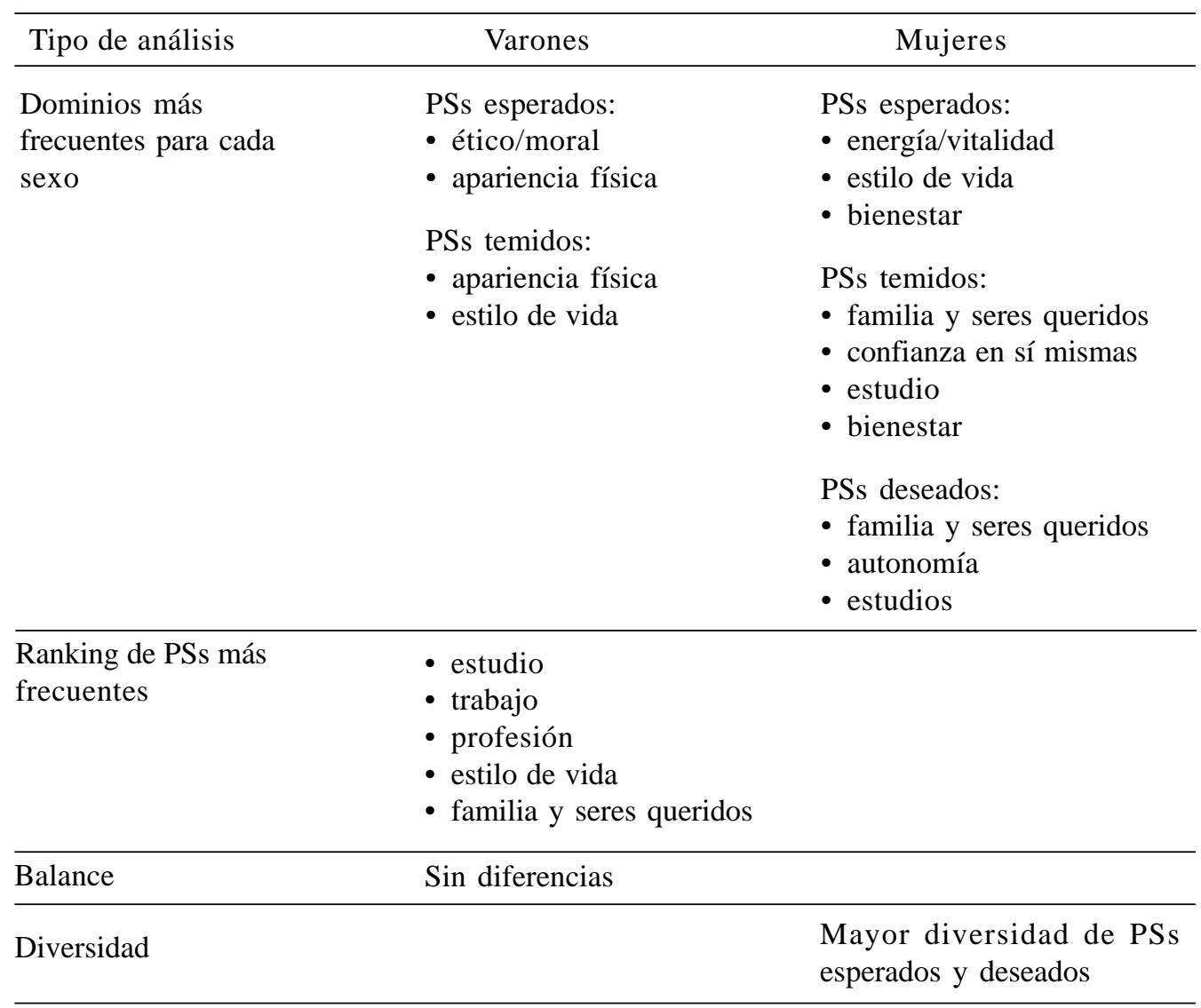

Nota: PSs = Posibles sí mismos. 


\section{Discusión}

El objetivo de este trabajo fue indagar las diferencias en función del sexo en aspectos de contenido, así como también en aspectos estructurales de los PSs de los adolescentes de zonas urbanas de Argentina. En primer lugar, consideramos la diferencia en la proporción en que los y las adolescentes generaron PSs en los diferentes dominios encontrados. Nuestros hallazgos muestran que, respecto de los PSs esperados, es mayor el porcentaje de varones que generaron PSs en el dominio ético/moral y apariencia física, y de mujeres que generaron PSs en los dominios energía/vitalidad, estilo de vida y bienestar. En cuanto a los PSs temidos, las mujeres presentan una mayor tendencia que los varones a proyectarse en los dominios de la familia y seres queridos, el estudio, la confianza en sí mismas, y el bienestar. Estos resultados son consistentes con estudios previos que muestran que las adolescentes generan más PSs temidos sobre funcionamiento interpersonal (Anthis et al., 2004; Knox et al., 2000; McClelland, 2011; Shepard \& Marshall, 1999; Zhu \& Tse, 2016) y el estudio (Zhu \& Tse, 2016). Sin embargo, estudios previos no encontraron una mayor prevalencia de mujeres que generaran PSs en el dominio de la autoconfianza o el bienestar. Es más, estudios previos encontraron que eran los varones los que presentaban en mayor medida PSs de fracaso general e inferioridad (Knox et al., 2000; McClelland, 2011). Por otro lado, en nuestro estudio encontramos que los varones presentan una mayor tendencia a crear PSs temidos en los dominios de la apariencia física y el estilo de vida. Estos resultados coinciden con los hallazgos de Shepard y Marshall (1999) quienes también encontraron una mayor proporción de PSs temidos en el dominio del estilo de vida en los varones. Sin embargo, nuestros resultados difieren de los de otros estudios en los que se encontró que más varones que mujeres generaron más PSs sobre su ocupación (Knox et al., 2000; Zhu \& Tse, 2016) o comportamiento riesgoso (Zhu \& Tse, 2016).
Respecto de los PSs deseados, encontramos un mayor porcentaje de mujeres que generaron PSs en los dominios de autonomía, estudios, y familia y seres queridos. Estos resultados coinciden con los estudios que muestran que las mujeres presentan más PSs deseados en el dominio interpersonal (McClelland, 2011; Zhu \& Tse, 2016) y del estudio (Zhu \& Tse, 2016). Sin embargo, los estudios anteriores presentan un mayor número de varones que generan PSs en el dominio de la carrera profesional y el estilo de vida (Zhu \& Tse, 2016) o las posesiones (Shepard \& Marshall, 1999).

En segundo lugar, analizamos si existían diferencias en los dominios de los PSs más frecuentemente generados por los varones y las mujeres. Los resultados muestran gran coincidencia entre los sexos en las categorías más mencionadas: el estudio, el trabajo, la profesión, el estilo de vida, y la familia y seres queridos. Estos resultados coinciden parcialmente con estudios previos que muestran que tanto varones como mujeres formulaban PSs con más frecuencia en el dominio de la ocupación. Sin embargo, en dicho estudio solo eran las mujeres las que lo hacían con más frecuencia en el dominio de las relaciones interpersonales (McClelland, 2011). Nuestros hallazgos indican que las relaciones interpersonales, más específicamente, la familia y seres queridos ocupan un lugar similar en la jerarquía de PSs de los varones y las mujeres.

En tercer lugar, estudiamos si los y las adolescentes difieren en la diversidad de dominios de PSs que pueden generar. Nuestros resultados indican que las mujeres crearon PSs esperados y deseados en una mayor diversidad de dominios que los varones. No se observan diferencias en la diversidad de dominios de los PSs temidos. Estos resultados coinciden con estudios previos que indican que las adolescentes informaron un mayor número de PSs deseados que los varones (McClelland, 2011; Zhu \& Tse, 2016). Asimismo, esto es coherente con el hecho de que las adolescentes pueden crear autorrepresentaciones actuales en una mayor variedad de dominios (Harter, 1990). 
Por último, analizamos si existen diferencias en el balance entre los PSs esperados y los temidos. No se encontraron diferencias respecto del balance en los PSs. Nuestros resultados coinciden con los de Pavulens (2013). Sin embargo, difieren de los de Anthis et al. (2004), quienes encontraron diferencias en el balance en el dominio interpersonal. Es posible que esto se deba a que en dicho estudio se consideró el balance por dominio y en el nuestro se estudió el balance general en los PSs.

Entre los resultados mencionados, se destaca la baja frecuencia con que tanto los varones como las mujeres producen PSs en el dominio familiar. Más allá de que las mujeres informen con más frecuencia que los varones esta categoría para sus PSs temidos y deseados, esta categoría es mencionada con una baja frecuencia por ambos sexos. Al respecto, es importante considerar el punto de referencia temporal que propusimos a los adolescentes para proyectarse: el final de la secundaria. Según el observatorio de la maternidad (Lupita \& Cogliandro, 2013), en Argentina, la edad promedio de las mujeres al tener su primer hijo/a es de 24.4 años; es decir, seis años después de haber terminado la secundaria. Por otro lado, cabe destacar que trabajamos con adolescentes que asisten a escuelas privadas de la CABA. En CABA la edad promedio al tener el primer hijo es de 27.4 años y en hogares con mayores niveles de ingresos la edad es de 27.4 a 28 años. Asimismo, las madres que realizan estudios universitarios 0 terciarios retrasan casi en 6 años la maternidad respecto de las que no lo hacen (28.3 años vs. 22.3 años).

Por otra parte, es importante tener en cuenta que los padres son un modelo a partir del cual los adolescentes desarrollan sus PSs (Oyserman \& James, 2011). En esta muestra más del $60 \%$ de las madres y padres tienen estudios universitarios completos. Por otra parte, el 86\% de las madres tienen empleo estable. Esto es consistente con las categorías más frecuentemente mencionadas por los adolescentes: estudios, ocupación y trabajo y con que no haya diferencias en la frecuencia con que varones y mujeres construyan PSs con este contenido.
El objetivo de este trabajo fue estudiar los PSs de los adolescentes considerando las diferencias entre los sexos. Para ello se trabajó con un grupo que concurre a una escuela secundaria privada y católica de la Ciudad de Buenos Aires. Es importante que en futuros estudios se consideren estas diferencias entre los y las adolescentes de escuelas públicas, de escuelas laicas o de otras religiones o, incluso, adolescentes que no estén escolarizados.

Por otro lado, sería importante considerar el efecto moderador de la edad. El final de la secundaria no tiene el mismo significado para los adolescentes que están iniciando sus estudios que para los que los están terminándola. Esto lleva a preguntarse si los y las adolescentes que están en los primeros años del nivel secundario presentan las mismas diferencias en sus PSs que los que están en los últimos años.

A pesar de estas limitaciones, este estudio permitió describir las diferencias por sexo en los PSs de un grupo de adolescentes con características socioculturales particulares. Los resultados encontrados nos permiten conocer cómo los y las adolescentes se imaginan a sí mismos en un momento particular de su futuro: el final de la escolaridad. Este conocimiento es de particular importancia debido al alto efecto que tiene esta imagen futura en el comportamiento presente de los adolescentes. Los PSs se han asociado a una gran variedad de conductas saludables en la adolescencia como el compromiso con la escuela y el estudio (e.g., Destin \& Oyserman, 2010; Leondari, Gonida, \& Gialamas, 2009; Oyserman, Brickman, \& Rhodes, 2007). Asimismo, se asocian a menor incursión en conductas de riesgo como comportamiento violento (Pierce, Schmidt, \& Stoddard, 2015) o abuso de sustancias (Aloise-Young, Hennigan, \& Leong, 2001; Lee et al., 2015). Por este motivo se los considera una pieza clave para el trabajo con los adolescentes en distintos ámbitos. En el ámbito educativo y comunitario, el trabajo con los adolescentes en el desarrollo de sus PSs y de estrategias para lograrlos ha demostrado promover un mayor sentido de pertenencia en la escuela y 
preocupación por tener un buen desempeño académico, disminución de los problemas de conducta en la escuela, tener mayor asistencia, mayor participación en clase y tiempo dedicado a las tareas escolares; asimismo, disminuyó el riesgo de depresión (Oyserman, Bybee, \& Terry, 2006; Oyserman, Terry, \& Bybee, 2002). En el ámbito clínico, la exploración de PSs promueve la motivación para el cambio y el logro de los objetivos terapéuticas (Bak, 2015).

En la actualidad, la adolescencia yo no es pensada como etapa de patología y desviación, sino como una etapa llena de potencial. Esto implica la necesidad de alentar a los adolescentes a buscar alternativas diversas y validarlas (Molina, Raimundi, \& Gimenez, 2017). Durante la adolescencia y la adultez emergente, los jóvenes deben ser animados a participar en una búsqueda amplia y variada de propósito (Bronk, Hill, Lapsley, Talib, \& Finch, 2009). Los adolescentes necesitan oportunidades para lograr desafíos, explorar y consolidar su identidad, incrementar su autonomía y obtener experiencias necesarias para los roles adultos (Barber, Abbott, Blomfield, \& Eccles, 2009). Por lo tanto, los hallazgos de este estudio nos permiten pensar cómo se puede favorecer a cada género para el desarrollo de PSs que promuevan el bienestar, faciliten la exploración y formación de la identidad, y la elección de conductas que favorezcan un desarrollo positivo.

\section{Conclusiones}

Considerando los resultados en su conjunto, puede observarse que tanto los varones como las mujeres se proyectan a sí mismos al final del nivel secundario, principalmente, en el dominio de los estudios, la ocupación y el trabajo. Las mujeres tienen una mirada más amplia de sí mismas en el futuro, es decir, generan PSs en una mayor diversidad de dominios. Esto está relacionado con el hecho de que presenten en mayor medida que los varones PSs en dominios poco frecuentes como los de autonomía, bienestar, energía-vitalidad, confianza en sí mismas. Por otro lado, sigue siendo más prevalente para las mujeres la proyección de sí mismas en el dominio de la familia y los seres queridos.

\section{Conflicto de intereses}

No existe ningún conflicto de interés moral, económico, laboral o de investigación para la publicación de este manuscrito.

\section{Responsabilidad ética}

La participación fue anónima en todo el proceso, únicamente los investigadores tuvieron acceso a los datos. En este estudio no aparecen datos personales de los participantes.

\section{Contribución de autoría}

MFM: concepción y diseño del estudio, recolección de datos, análisis de los datos, redacción del manuscrito.

MB: análisis de los datos, redacción del manuscrito.

AA: análisis de los datos, redacción del manuscrito.

\section{Agradecimientos}

Este estudio fue financiado por la Universidad Nacional de Tres de Febrero (UNTREF). Proyecto: «Percepción de un futuro significativo en la adolescencia y su relación con el autoconcepto y el contexto familiar». Se agradece a los alumnos, docentes y autoridades de las escuelas en la que se realizó el estudio, su colaboración en este proyecto de investigación.

\section{Referencias}

Aloise-Young, P. A., Hennigan, K. M., \& Leong, C. W. (2001). Possible Selves and Negative Health Behaviors During Early Adolescence. The Journal of Early Adolescence, 21(2), 158-181. doi: 10.1177/02724 31601021002002

Anderman, E. M., Anderman, L. H., \& Griesinger, T. (1999). The Relation of Present and Possible Academic Selves During Early Adolescence to Grade Point 
Average and Achievement Goals. The Elementary School Journal, 100(1), 3-17. doi: 10.1086/461940

Anthis, K. S., Dunkel, C. S., \& Anderson, B. (2004). Gender and identity status differences in late adolescents' possible selves. Journal of Adolescence, 27(2), 147-152. doi: 10.1016/j.adolesc ence.2003.11.010

Bak, W. (2015). Possible Selves: Implications for Psychotherapy. International Journal of Mental Health and Addiction, 13(5), 650-658. doi: 10.1007/ s11469-015-9553-2

Barber, B. L., Abbott, B., Blomfield, C., \& Eccles, J. (2009). Secrets of Their Success: Activity Participation and Positive Youth Development. In R. Gilman, E. S. Huebner, \& M. J. Furlong (Eds.), Handbook of Positive Psychology in Schools ( $1^{\text {st }}$ ed., pp. 273-287). New York: Taylor \& Francis.

Bronk, K. C., Hill, P. L., Lapsley, D. K., Talib, T. L., \& Finch, H. (2009). Purpose, hope, and life satisfaction in three age groups. The Journal of Positive Psychology, 4(6), 500-510. doi: 10.1080/1743976 0903271439

Cadely, H. S.-E., Pittman, J. F., Kerpelman, J. L., \& AdlerBaeder, F. (2011). The Role of Identity Styles and Academic Possible Selves on Academic Outcomes for high School Students. Identity: An International Journal of Theory and Research, 11(4), 267-288. doi: 10.1080/15283488.2011.613580

Cohen, J. (1992). A power primer. Psychological Bulletin, 112(1), 155-159. doi: 10.1037/00332909.112.1.155

Cross, S. E., \& Madson, L. (1997). Models of the self: Self-construals and gender. Psychological Bulletin, 122(1), 5-37. doi: 10.1037/0033-2909.122.1.5

Destin, M., \& Oyserman, D. (2010). Incentivizing education: Seeing schoolwork as an investment, not a chore. Journal of Experimental Social Psychology, 46(5), 846-849. doi: 10.1016/j.jesp.2010.04.004

Facio, A., Resett, S., Braude, M., \& Benedetto, N. (2006). El Perfil de Autopercepción para Adolescentes de Paraná, Buenos Aires y Río Gallegos [Self-Perception Profile for Adolescents in youth from Paraná, Buenos
Aires and Río Gallegos]. Investigaciones en Psicología, 11(3), 7-25.

Halfond, R., Corona, R., \& Moon, A. (2013). Latino Parent and Adolescent Perceptions of Hoped-for and Feared Possible Selves for Adolescents. Journal of Adolescent Research, 28(2), 209-240. doi: 10.1177/ 0743558412457818

Harter, S. (1990). Developmental differences in the nature of self-representations: Implications for the understanding, assessment, and treatment of maladaptive behavior. Cognitive Therapy and Research, 14(2), 113-142. doi: 10.1007/BF01176205

Harter, S. (2012). Self-perception Profile for Adolescents: Manual and questionnaires revision. Denver, CO: University of Denver.

Josephs, R. A., Markus, H., \& Tafarodi, R. W. (1992). Gender and self-esteem. Journal of Personality and Social Psychologyersonality And, 63(3), 991-402. doi: 10.1037/0022-3514.63.3.391

Knox, M., Funk, J., Elliott, R., \& Bush, E. G. (2000). Gender differences in adolescents' possible selves. Youth \& Society, 31(3), 287-309. doi: 10.1177/ 0044118X00031003002

Lee, C. K., Corte, C., Stein, K. F., Park, C. G., Finnegan, L., \& Mccreary, L. L. (2015). Prospective Effects of Possible Selves on Alcohol Consumption in Adolescents. Research in Nursing and Health, 38(1), 71-81. doi: 10.1002/nur.21641

Leondari, A., Gonida, E. N., \& Gialamas, V. (2009). 'Possible selves' during middle adolescence: Relationships with school achievement and with various demographic factors. The Journal of the Hellenic Psychological Society, 16(4), 342-360.

Leondari, A., Syngollitou, E., \& Kiosseoglou, G. (1998). Academic Achievement, Motivation and Future Selves. Educational Studies, 24(2), 153-163. doi: 10.1080/ 0305569980240202

Lips, H. M. (2004). The Gender Gap in Possible Selves: Divergence of Academic Self-Views Among High School and University Students. Sex Roles, 50(5-6), 357-371. doi: 10.1023/B:SERS.0000018891.888 89.c9 
Lupita, C., \& Cogliandro, G. (2013). Cuadernillo estadístico de la maternidad $N^{\circ}$ 7. Maternidad en Argentina: aspectos demográficos, sociales, educativos y laborales: procesamiento de datos de la Encuesta Permanente de Hogares, INDEC. Periodo 2006-2012 (1er ed.). Buenos Aires, Argentina: Observatorio de la Maternidad. Recuperado de http://www.o-maternidad.org.ar/publicaciones/ cuadernillo/2013.aspx

Malin, H., Reilly, T. S., Quinn, B., \& Moran, S. (2014). Adolescent Purpose Development: Exploring Empathy, Discovering Roles, Shifting Priorities, and Creating Pathways. Journal of Research on Adolescence, 24(1), 186-199. doi: 10.1111/jora.12051

Markus, H., \& Nurius, P. (1986). Possible Selves. American Psychologist, 41(9), 954-969. doi: 10.1037/ 0003-066X.41.9.954

McClelland, C. (2011). An examination of the present and future selves of adolescents: Investigating possible selves and self-esteem in relation to gender and attachment. The Plymouth Student Scientist, 4(2), 104-130. Recuperado de https://pearl.plymouth.ac.uk/ handle/10026.1/13952

Molina, M. F., \& Raimundi, M. J. (2011). Predictores de la autoestima global en niños de escuela primaria de la Ciudad de Buenos Aires. Diferencias en función del sexo y la edad. Revista Argentina de Ciencias Del Comportamiento, 3(3), 1-7. Recuperado de http:// www.revistas.unc.edu.ar/index.php/racc/article/view/ 5201

Molina, M. F., Raimundi, M. J., \& Bugallo, L. (2017). La percepción de los estilos de crianza y su relación con las autopercepciones de los niños de Buenos Aires: Diferencias en función del género [Perceived parenting styles and their relation to self-perceptions of children from Buenos Aires]. Universitas Psychologica. Advance Online Publication, 16(1). doi: 10.11144/Javeriana.upsy16-1.pecr

Molina, M. F., Raimundi, M. J., \& Gimenez, M. (2017). Los posibles sí mismos de los adolescentes de Buenos Aires [The possible selves of adolescents from Buenos Aires]. Revista Latinoamericana de Ciencias Sociales, Niñez y Juventud [Latin American Journal in Social Sciences, Childhood and Youth], 15(1), 455-470. doi: 10.11600/1692715x.1512901102015
Molina, M. F., Raimundi, M. J., López, C., Cataldi, S., \& Bugallo, L. (2011). Adaptación del Perfil de Autopercepciones para Niños para su uso en la Ciudad de Buenos Aires [Adaptation of the SelfPerception Profile for Children for its Use in Buenos Aires City]. Revista Iberoamericana de Diagnóstico y Evaluación Psicológica, 2(32), 53-78.

Molina, M. F., Schmidt, V., \& Raimundi, M. J. (2017a). Adolescents Possible Selves and Parental Promotion of Challenges. In J. A. Sørensen \& N. S. Schultz (Eds.), Self-Perception: Research Advances and Clinical Challenges (pp. 83-125). New York: Nova Science.

Molina, M. F., Schmidt, V., \& Raimundi, M. J. (2017b). Possible Selves in Adolescence: Development and Validation of a Scale for their Assessment. Journal of Psychology: Interdisciplinary and Applied, 151(7), 646-668. doi: 10.1080/00223980.2017.1372347

Norman, C. C., \& Aron, A. (2003). Aspects of possible self that predict motivation to achieve or avoid it. Journal of Experimental Social Psychology, 39(5), 500-507. doi: 10.1016/S0022-1031(03)00029-5

Oyserman, D. (2004). Possible selves citations, measure, and coding instructions. Ann Arbor, MI: University of Michigan. Recuperado de https://dornsife.usc.edu/ assets/sites/782/docs/possible_selves_measure.doc

Oyserman, D., Brickman, D., \& Rhodes, M. (2007). School Success, Possible Selves, and Parent School Involvement. Family Relations, 56(5), 479-489. doi: 10.1111/j.1741-3729.2007.00475.x/full

Oyserman, D., Bybee, D., \& Terry, K. (2006). Possible selves and academic outcomes: How and when possible selves impel action. Journal of Personality and Social Psychology, 91(1), 188-204. doi: 10.1037/ 0022-3514.91.1.188

Oyserman, D., Bybee, D., Terry, K., \& Hart-Johnson, T. (2004). Possible selves as roadmaps. Journal of Research in Personality, 38(2), 130-149. doi: 10.101 6/S0092-6566(03)00057-6

Oyserman, D., \& Fryberg, S. (2006). The possible selves of diverse adolescents: Content and function across gender, race and national origin. In C. Dunkel \& J. Kerpelman (Eds.), Possible selves: Theory, Research, and Applications (pp. 1-23). New York: Nova Science Publishers. 
Oyserman, D., \& James, L. (2009). Possible selves: From content to process. In K. D. Markman, W. M. Klein, \& J. A. Suhr (Eds.), Handbook of Imagination and Mental Simulation (pp. 373-394). New York: Taylor \& Francis.

Oyserman, D., \& James, L. (2011). Possible identities. In S. J. Schwartz, K. Luyckx, \& V. L. Vignoles (Eds.), Handbook of Identity Theory and Research (pp. 117145). New Jersey: Springer Link. doi: 10.1007/9781-4419-7988-9

Oyserman, D., Terry, K., \& Bybee, D. (2002). A possible selves intervention to enhance school involvement. Journal of Adolescence, 25, 313-326. doi: 10.1006/ yjado. 474

Pavulens, J. (2013). Career-related possible selves of rural adolescents. Research for Rural Development, 2, 221228. Recuperado de http://www2.llu.lv/research_conf/ Proceedings/19th_volume2.pdf

Pierce, J., Schmidt, C., \& Stoddard, S. A. (2015). The role of feared possible selves in the relationship between peer influence and delinquency. Journal of Adolescence, 38, 17-26. doi: 10.1016/j.adolescence. 2014.10.009

Programa de las Naciones Unidas para el Desarrollo (2018). Índices e indicadores de desarrollo humano. Actualización estadística de 2018. Nueva York: Communications Development Incorporated.

Resett, S., Meier, L., García-Sánchez, O., \& Katz, O. (2016). Predicción del autoconcepto a partir de las emociones maternas en niños de edad escolar y diferencias según sexo [Prediction of self-concept in children by maternal emotion and sex differences]. Psicodebate, 16(1), 27-48. doi: 10.18682/pd.v16 i1. 565
Shepard, B., \& Marshall, A. (1999). Possible Selves Mapping: Life-Career Exploration with Young Adolescents. Canadian Journal of Counselling, 33(1), 37-54. Recuperado de http://eric.ed.gov/ ?id=EJ587281

Sica, L. S. (2009). Adolescents in different contexts: The exploration of identity through possible selves. Cognition, Brain, Behavior. An Interdisciplinary Journal, 13(3), 221-252. Recuperado de https://psyc net.apa.org/record/2009-21493-001

Strauss, A., \& Corbin, J. (1990). Basics of qualitative research: Grounded theory procedures and techniques. Thousand Oaks, CA, US: SAGE Publications, Inc.

Unemori, P., Omoregie, H., \& Markus, H. R. (2004). Self-Portraits: Possible Selves in EuropeanAmerican, Chilean, Japanese and Japanese-American Cultural Contexts. Self and Identity, 3(4), 321-338. doi: 10.1080/13576500444000100

Yowell, C. M. (2002). Dreams of the Future: The Pursuit of Education and Career Possible Selves Among Ninth Grade Latino Youth. Applied Developmental Science, 6(2), 62-72. doi: 10.1207/S1532480XA DS0602_2

Zhu, S., \& Tse, S. (2016). Possible selves, strategies and perceived likelihood among adolescents in Hong Kong: desire and concern. International Journal of Adolescence and Youth. Advance on Line Publication., 21(2), 135-149. doi: 10.1080/0267384 3.2015 .1031683 


\section{María Fernanda Molina}

Universidad Nacional de Tres de Febrero (UNTREF), Argentina

Doctora en Psicología. Jefa de trabajos prácticos de la materia Metodología de la investigación de la Licenciatura en Psicomotricidad de UNTREF. Su línea de investigación está centrada en temas relacionados a autopercepciones infanto-juveniles.

ORCID: 0000-0002-7361-7058

Autor corresponsal: fmolina@untref.edu.ar,fer_molina@hotmail.com

\section{Melisa Benzi}

Universidad de Buenos Aires, Argentina

Licenciada en Psicología. Integrante del Proyecto de Investigación de la Secretaría de Ciencia y Técnica de la Universidad de Buenos Aires «Consumo de alcohol en población joven: factores protectores a través del deporte y la música». Línea de investigación: autopercepciones en jóvenes, realización personal y disfrute.

ORCID: 0000-0002-1889-5231

melisafernandezben@gmail.com

\section{Analía Álvarez}

Universidad de Buenos Aires, Argentina

Licenciada en Psicología. Integrante del Proyecto de Investigación de la Secretaría de Ciencia y Técnica de la Universidad de Buenos Aires «Consumo de alcohol en población joven: factores protectores a través del deporte y la música». Línea de investigación: autopercepciones en jóvenes, disfrute en el deporte.

ORCID: 0000-0003-4301-4165

licalvarez@msn.com 